\title{
Les dystrophies maculaires de la rétine
}

Depuis cinq ans la génétique a acquis droit de cité dans le domaine des affections de la rétine, mais il reste encore bien du chemin à parcourir. Pour embrasser l'ensemble des phénomènes, il faut se rappeler que la rétine est irriguée par un riche réseau vasculaire issu de la choroïde ; entre rétine et choroïde s'intercale une couche de cellules pigmentées épithéliales ; entre cette couche et la choroïde se trouve une matrice extracellulaire, la membrane de Bruch, formée de protéines de structure comme le collagène, la laminine et la fibronectine.

Parmi les maladies de la rétine, les mieux étudiées jusqu'ici ont été les rétinites pigmentaires. De nombreux mutants y ont été découverts; ils ont d'abord intéressé la rhodopsine, pigment des photorécepteurs des bâtonnets, dont le gène est sur le chromosome $3\left(\mathrm{~m} / \mathrm{s} n^{\circ} 4\right.$, vol. $6, p$. 402 et $n^{\circ} 1$, vol. 8, p. 82), puis la périphérine (gène sur le chromosome 6$)(\mathrm{m} / \mathrm{s}$ $n^{\circ} 2$, vol. $8, p$. 171 et $n^{\circ} 4$, vol. 9, p. 478). Des modèles animaux ont été également reconnus, tels, chez la souris, retinal degeneration (périphérine) et retinal degeneration-slow (sousunité alpha de la phosphodiestérase du GMP cyclique).

Récemment, l'attention s'est portée sur le groupe des dystrophies maculaires. La macula est la partie centrale, riche en cônes, de la rétine. Sa dégénérescence entraîne la perte de la vision centrale. On en connaît des formes familiales, et c'est parmi elles qu'on a découvert des anomalies de la périphérine. Cependant, celles-ci restent très minoritaires dans l'ensemble des dystrophies maculaires. De loin les plus nombreux cas sont fournis par des formes plus tardives, qualifiées de dégénérescences maculaires liées à l'âge. C'est là une cause de cécité très fréquente, atteignant environ une personne sur 40. On pense que le milieu joue un rôle dans leur apparition, mais il existe des dispositions familiales certaines ; on tend à en faire des maladies multigéniques, cependant des formes monogéniques simples peuvent être reconnues ; parmi celles-ci, on a individualisé un syndrome rare, la dystrophie du fond d'œil de Sorsby, affection autosomique dominante atteignant des sujets de 30 à 40 ans. Elle est marquée par des dépôts jaunâtres dans la membrane de Bruch, entravant le développement et le fonctionnement des capillaires. La matrice intercellulaire contient une série de métalloprotéinases, dont le fonctionnement est soumis à un contrôle par des inhibiteurs tissulaires (TIMP) dont on pense que des anomalies pourraient être impliquées dans des maladies dégénératives. Récemment l'un d'entre eux, TIMP3, a été identifié et son gène localisé sur le chromosome 22 en 22q12.1-q13.2 [1, 2]. Un groupe de Wurzburg (Allemagne), étudiant la dystrophie de Sorsby, a localisé son locus dans cette même région [3]. Il a dès lors entrepris de vérifier si TIMP3 n'était pas responsable de la maladie. L'ADNc de TIMP3 a été cloné $[1,2]$. Le gène s'étend sur environ $20 \mathrm{kpb}$, compte 5 exons et code pour une protéine de 188 acides aminés ; il existe un peptide signal. Dans deux familles atteintes on trouva une mutation dans l'exon 5: une transition $A \rightarrow G$ change une Tyr en Cys au codon 168 ; une transversion $\mathrm{A} \rightarrow \mathrm{T}$ change une Ser en Cys au codon 181 [4]. Ces mutations sont absentes chez les membres sains de ces familles et chez les témoins. Dans les deux cas, l'acide aminé normal est conservé au cours de l'évolution, et, surtout, celui qui apparaît est une cystéine. Or la caractéristique majeure de la molécule (commune d'ailleurs à tous les TIMP connus) est la présence de 12 cystéines, arrangées probablement en 6 ponts disulfure ; l'apparition d'une cystéine surnuméraire risque donc de créer, soit un groupe SH isolé susceptible de se combiner avec son homologue d'une autre molécule, soit de modifier la nature des ponts disulfure à l'intérieur de la molécule et d'en changer la conformation. La modification d'activité du
TIMP3 peut ainsi entraîner un changement dans l'action des protéinases, normalement soumises au contrôle de l'inhibiteur.

Reste une question, peut-être la plus importante [5]. Pour intéressante qu'elle soit, la mutation du TIMP3 n'intervient que dans une proportion très faible des dystrophies maculaires. D'autres déficits monogéniques existent probablement, en partie masqués par la date tardive de la révélation de la plupart des affections rétiniennes. Mais il est vraisemblable que les formes polygéniques sont les plus nombreuses. Leur détection sera nécessairement difficile. On peut rappeler ici $\left(m / s n^{\circ} 8-9\right.$, vol. 10 , p. 908) qu'une forme de rétinite pigmentaire a pu être rapportée au déficit conjugué de deux protéines non génétiquement liées, la périphérine et ROM1, dont aucune des anomalies ne provoquait séparément de troubles. Cette maladie "digénique» serait un premier exemple, que pourraient suivre d'autres maladies mettant en cause diverses métalloprotéines et leurs inhibiteurs. Une consêquence heureuse qu'aurait l'élucidation de ces maladies polygéniques serait qu'une thérapie génique agissant sur un seul des facteurs de l'affection pourrait suffire à améliorer l'ensemble de la symptomatologie.

J.C.D.

1. Apte SS, Mattei MG, Olsen BR. Cloning of the cDNA encoding human tissue inhibitor of metalloproteinase-3 (TIMP-3) and mapping of the TIMP-3 gene to chromosome 22. Genomics 1994 ; $19: 86-90$.

2. Silbiger SM, Jacobsen VL, Cuppies RL, Koski RA. Cloning of cDNAs encoding TIMP-3, a novel member of the tissue inhibitor of metalloproteinase family. Gene 1994 ; 141 : 293-7.

3. Weber BHF, Vogt G, Wolz W, lves EJ, EwingCC. Sorsby's fundus dystrophy is genetically linked to chromosome 22 q 13-qter. Nalure Genet $1994 ; 7$ : 158-61.

4. Weber BHV, Vogt G, Pruett RC, Stohr H, Felbor U. Mutations in the tissue inhibitor of metalloproteinases-3 (TIMP3) in patients with Sorsby's fundus dystrophy. Nature Genet $1994 ; 8: 352-6$.

5. Humphries P, Kenna P, Farrar GJ. New dimensions in macular dystrophies. Nature Genet 1994 ; 8: 315-7. 